\title{
Effects of Eicosapentaenoic Acid on Platelet Function in Patients Taking Long-Term Aspirin Following Coronary Stent Implantation
}

\author{
Kaoru Takada, ${ }^{1} \mathrm{MD}$, Shuichi Ishikawa, ${ }^{1} \mathrm{MD}$, Naoyuki Yokoyama, ${ }^{1} \mathrm{MD}$, \\ Naoyoshi Hosogoe, ${ }^{1} \mathrm{MD}$, and Takaaki Isshiki, ${ }^{1} \mathrm{MD}$
}

\begin{abstract}
SUMMARY
Eicosapentaenoic acid (EPA) has been widely accepted to have antiatherosclerotic effects. The aim of this study was to investigate the antiplatelet effect of EPA combined with acetylsalicylic acid (ASA) following stent implantation. Eighteen patients who had undergone coronary stent implantation at least 8 months previously were included. All patients were given EPA ethyl ester (EPA-E) $1.8 \mathrm{~g} /$ day in addition to ASA $100 \mathrm{mg} /$ day for 12 weeks. After the treatment, the plasma EPA/arachidonic acid (AA) ratio increased significantly from $0.40 \pm 0.2$ to $1.08 \pm 0.39(P<0.001)$. There were no changes in the maximum platelet aggregation (MPA) induced by adenosine diphosphate ( 5 and $20 \mu \mathrm{mol} / \mathrm{L}), \mathrm{AA}$ $(0.3$ and $0.5 \mathrm{mg} / \mathrm{mL})$, or collagen $(2$ and $4 \mu \mathrm{g} / \mathrm{mL})$. Furthermore, no significant differences were observed in the expression of PAC-1 and CD62P on the platelet surface membranes or in the soluble P-selectin concentration. With further analysis, a significant negative correlation was found between collagen $(2 \mu \mathrm{g} / \mathrm{mL})$-induced MPA and plasma EPA/AA ratio $(r=-0.507, P=0.032$ ). The patients were then divided into 2 groups according to the median EPA/AA ratio value of 0.92. In the high EPA/AA ratio group $(n=10)$, collagen-induced MPA was significantly suppressed after EPA-E administration $(45.3 \pm 15.9$ versus $39.0 \pm 16.3, P=0.033)$. In contrast, there were no significant changes in platelet aggregation $(56.0 \pm 9.8$ versus $57.1 \pm 11.4, P=0.745)$ in the low EPA/AA ratio group $(n=8)$. EPA treatment had a potential to suppress collagen-induced platelet aggregation in patients with a high plasma EPA/AA ratio. (Int Heart J 2014; 55: 228-233)
\end{abstract}

Key words: Omega-3 polyunsaturated fatty acids, Coronary artery disease

$\mathrm{C}$ oronary revascularization with drug-eluting stents (DES) has become a widespread technique for treating coronary artery disease (CAD). The guidelines recommend that patients with DES should receive dual antiplatelet therapy (DAPT) with acetylsalicylic acid (ASA) and thienopyridine for at least 1 year in order to prevent stent thrombosis. ${ }^{1,2)}$ When the risk of stent thrombosis decreases in the chronic phase following DES implantation, secondary prevention becomes a primary target of patient care. In fact, in the chronic phase, the risks of CAD or cerebrovascular disease are much higher than that of stent thrombosis. ${ }^{3,4)}$ Therefore, lifelong treatment with ASA is also recommended for the secondary prevention of atherothrombotic events following DAPT termination. ${ }^{1,2)}$

Eicosapentaenoic acid (EPA) has been widely accepted to have antiatherosclerotic effects. ${ }^{5,6}$ In relation to secondary prevention, the use of eicosapentaenoic acid ethyl ester (EPA-E) in combination with statins successfully reduced the recurrence of cardiovascular events in the Japan EPA lipid intervention study (JELIS). ${ }^{7)}$ In particular, in patients who underwent coronary artery intervention, the JELIS sub-study showed that the incidence of major coronary events was significantly re- duced by $41 \%$ in the EPA treatment group. ${ }^{8}$ EPA is known to improve vascular endothelial function ${ }^{9)}$ and to reduce platelet function ${ }^{10)}$ These multi-functional characteristics of EPA may have contributed to the positive results of the JELIS study, in which EPA-E was used concomitantly with ASA in the majority of the JELIS patients who had histories of atherothrombotic events. Therefore, it is important to know how platelet function is affected in patients receiving combination therapy of ASA and EPA. However, only a few studies have reported an additional effect of EPA on platelet function in patients with CAD.

In this study, we investigated the effects of EPA-E on platelet aggregation and platelet activation in patients under ASA treatment after coronary stent implantation.

\section{Methods}

Study population: Twenty male patients with stable CAD were recruited for this study. Eligible patients, who were taking statins for dyslipidemia, had undergone coronary stent implantation at least 8 months previously and were placed on continuous ASA (100 mg/day) administration after stent im-

From the ${ }^{1}$ Division of Cardiology, Department of Internal Medicine, Teikyo University School of Medicine, Tokyo, Japan.

Address for correspondence: Takaaki Isshiki, MD, Division of Cardiology, Department of Internal Medicine, Teikyo University School of Medicine, 2-11-1 Kaga Itabashi-ku, Tokyo 173-8605 Japan. E-mail: isshiki@med.teikyo-u.ac.jp

Received for publication October 2, 2013. Revised and accepted November 19, 2013

Released advance online J-STAGE May 7, 2014.

All rights are reserved to the International Heart Journal Association. 
plantation. Exclusion criteria were as follows: age $<50$ or $>75$ years, familial hyperlipidemia, liver injury (AST $\geq 100 \mathrm{U} / \mathrm{L}$, ALT $\geq 100 \mathrm{U} / \mathrm{L}, \gamma \mathrm{GTP} \geq 150 \mathrm{U} / \mathrm{L}$, ALP $\geq 300$, total bilirubin $\geq$ 3 ), renal insufficiency (serum creatinine $\geq 2 \mathrm{mg} / \mathrm{dL}$ ), recent bleeding episode, uncontrollable hypertension (systolic $\mathrm{BP} \geq$ $180 \mathrm{mmHg}$ or diastolic BP $\geq 110 \mathrm{mmHg}$ ), diabetes mellitus with $\mathrm{HbA} 1 \mathrm{c} \geq 10 \%$, scheduled for surgery, use of nonsteroidal anti-inflammatory drugs, and previous treatment with antiplatelet therapy other than ASA within the past 30 days. This study was approved by the ethics committee of Teikyo University Hospital (No.11-095), and written informed consent was obtained from all patients included in the study.

Study design: All patients were treated with EPA-E at a dose of $1.8 \mathrm{~g} /$ day (900 mg, twice per day after meals) for 12 weeks. All patients also received ASA at a dose of $100 \mathrm{mg}$ daily. The patients who were taking statin were not allowed to change the dose during the study period. Indexes of platelet function were evaluated before and after EPA-E administration by determining the levels of platelet aggregation induced by adenosine diphosphate (ADP), collagen, and arachidonic acid (AA) in platelet-rich plasma (PRP) evaluating platelet surface expression levels of activation markers such as PAC-1 and CD62P (P-selectin) and measuring soluble P-selectin concentration. Additionally, levels of plasma EPA, AA, total cholesterol (T$\mathrm{CHO}$ ), high-density lipoprotein cholesterol (HDL-C), lowdensity lipoprotein cholesterol (LDL-C), and triglycerides (TG) were also measured before and after EPA-E administration.

Blood sampling: Blood samples for platelet function assays were obtained from an antecubital vein using a 21-gauge needle. To avoid spontaneous platelet activation, the first 4 to 5 $\mathrm{mL}$ of blood was used for clinical chemistry analysis. Additionally, the blood used for measuring soluble P-selectin was centrifuged for 10 minutes at $3000 \mathrm{rpm}$. The plasma was then frozen and stored at $-20^{\circ} \mathrm{C}$ until analysis.

Platelet aggregation assessment: Maximum platelet aggregation (MPA) was assessed by light transmittance aggregometry (LTA) with an MCM HEMA-TRACER 212 aggregometer (MC Medical, Tokyo) after stimulation with 5 or $20 \mu \mathrm{mol} / \mathrm{L}$ ADP, 2 or $4 \mu \mathrm{g} / \mathrm{mL}$ collagen, or 0.3 or $0.5 \mathrm{mg} / \mathrm{mL}$ AA. The maximum platelet aggregation rate (MPR) curves were recorded for 5 minutes. PRP was obtained as a supernatant after centrifugation of citrated blood at $1000 \mathrm{rpm}$ for 10 minutes. Isolated PRP was kept at room temperature before use. Plateletpoor plasma (PPP) was obtained by a second centrifugation of the blood fraction at $3000 \mathrm{rpm}$ for 15 minutes. Light transmission was adjusted to $0 \%$ with PRP and $100 \%$ with PPP for each measurement. Platelet aggregation was determined as the maximum percentage change in light transmittance from baseline, using PPP as a reference.

Platelet activation assessment: Platelet activation was assessed by flow cytometry (BD FACS Calibur flow cytometer; BD Bioscience, San Jose, CA, USA), determined by evaluating the platelet surface expression levels of PAC-1 (monoclonal antibody specific for fibrinogen receptors expressed on GPIIb/IIIa of the surface membranes of activated platelets) and CD62P (glycoprotein on the alpha-granule membranes that is exposed on the surface membranes when platelets are activated). Platelet activation was expressed as the percentage of platelets positive for MoAb binding. The soluble P-selectin concentration was measured using a GMP-140 (P-selectin) EIA kit (Takara,
Kyoto, Japan) on the basis of a sandwich enzyme-linked immunosorbent assay, according to the manufacturer's protocol. Statistical analysis: Continuous variables are expressed as the mean \pm standard deviation (SD), and the values obtained before and after EPA-E administration were compared using the paired $t$-test. Associations between continuous variables were tested at ad hoc basis with multivariate linear regression analysis using the forced-entry method, producing standardised partial regression coefficient $(\beta)$. Categorical variables are expressed as frequencies and percentages. Differences were considered significant at $P<0.05$ (two-tailed). All statistical analyses were performed using SPSS 17.0 software (SPSS Inc., Chicago, IL).

\section{RESULTS}

Twenty patients were recruited from January 2010 to May 2011. According to a questionnaire survey, 2 of the 20 study patients were found to be noncompliant in taking ASA, and were excluded from the study. Thus, a total of 18 patients were finally selected for analysis. Baseline demographic and clinical characteristics of the study patients are listed in Table I. Changes in plasma fatty acids and lipid levels: After 12 weeks of EPA-E treatment, the plasma EPA concentration increased and the plasma AA concentration decreased significantly. Accordingly, the ratio of EPA to AA (EPA/AA) increased significantly. Plasma T-CHO, LDL-C, and TG levels decreased significantly; however, the HDL-C level did not change (Table II). Effect of EPA-E on platelet functions: Platelet count and the other blood counts did not change following EPA administration. There were no significant changes in the MPA induced by ADP ( 5 and $20 \mu \mathrm{mol} / \mathrm{L}), \mathrm{AA}(0.3$ and $0.5 \mathrm{mg} / \mathrm{mL})$, or collagen (2 and $4 \mu \mathrm{g} / \mathrm{mL}$ ). Furthermore, no significant differences were

Table I. Baseline Characteristics of Study Patients

\begin{tabular}{lc}
\hline & $n=18$ \\
\hline Age (years) & $63.6 \pm 7.9$ \\
Male & $18 / 18(100 \%)$ \\
BMI $\left(\mathrm{kg} / \mathrm{m}^{2}\right)$ & $25.6 \pm 2.5$ \\
Dyslipidemia & $18 / 18(100 \%)$ \\
Hypertension & $14 / 18(77.8 \%)$ \\
Diabetes & $5 / 18(27.8 \%)$ \\
PAD & $0 / 18(0 \%)$ \\
Smoking & $8 / 18(44.4 \%)$ \\
\hline
\end{tabular}

Table II. Changes in Blood Count, Plasma Fatty Acids and Lipid Levels Before and After EPA-E Administration

\begin{tabular}{lccr}
\hline & Pre & 3 Mo & $P$ \\
\hline PLT $\left(\times 10^{4} / \mu \mathrm{l}\right)$ & $20.73 \pm 5.14$ & $21.28 \pm 5.25$ & 0.271 \\
$\mathrm{WBC}\left(\times 10^{2} / \mu \mathrm{l}\right)$ & $63.50 \pm 25.43$ & $61.44 \pm 17.64$ & 0.686 \\
$\mathrm{Hb}(\mathrm{g} / \mathrm{dL})$ & $14.01 \pm 1.10$ & $14.17 \pm 1.20$ & 0.234 \\
& & & \\
$\mathrm{~T}-\mathrm{CHO}(\mathrm{mg} / \mathrm{dL})$ & $161.00 \pm 24.5$ & $150.67 \pm 22.4$ & 0.004 \\
$\mathrm{HDL}-\mathrm{C}(\mathrm{mg} / \mathrm{dL})$ & $51.67 \pm 12.8$ & $48.67 \pm 10.9$ & 0.611 \\
$\mathrm{LDL}-\mathrm{C}(\mathrm{mg} / \mathrm{dL})$ & $82.06 \pm 21.9$ & $75.61 \pm 20.5$ & 0.034 \\
$\mathrm{TG}(\mathrm{mg} / \mathrm{dL})$ & $160.61 \pm 132.7$ & $149.44 \pm 74.1$ & 0.047 \\
EPA $(\mu \mathrm{g} / \mathrm{mL})$ & $67.24 \pm 32.0$ & $147.81 \pm 45.0$ & $<0.001$ \\
$\mathrm{AA}(\mu \mathrm{g} / \mathrm{mL})$ & $181.03 \pm 54.6$ & $144.13 \pm 35.3$ & $<0.001$ \\
EPA/AA ratio & $0.40 \pm 0.20$ & $1.08 \pm 0.39$ & $<0.001$ \\
\hline
\end{tabular}


Table III. Comparisons of Platelet Function Before and After EPA-E Administration

\begin{tabular}{lccc}
\hline & Pre & 3Mo & $P$ \\
\hline MPR $(\%)(n=18)$ & & & \\
ADP $5 \mu \mathrm{M}$ & $66.94 \pm 4.50$ & $66.72 \pm 4.36$ & 0.795 \\
ADP $20 \mu \mathrm{M}$ & $71.72 \pm 4.30$ & $72.50 \pm 3.17$ & 0.474 \\
Collagen $2 \mu \mathrm{g} / \mathrm{mL}$ & $50.06 \pm 14.29$ & $47.06 \pm 16.74$ & 0.183 \\
Collagen $4 \mu \mathrm{g} / \mathrm{mL}$ & $65.67 \pm 11.57$ & $63.00 \pm 12.28$ & 0.122 \\
Arachidonic acid $0.3 \mathrm{mg} / \mathrm{mL}$ & $4.11 \pm 1.53$ & $3.72 \pm 1.53$ & 0.233 \\
Arachidonic acid $0.5 \mathrm{mg} / \mathrm{mL}$ & $4.50 \pm 1.58$ & $4.50 \pm 1.86$ & 1.000 \\
Expression of platelet surface $(\%)(n=17)$ & & & \\
PAC1 & $15.56 \pm 11.80$ & $13.03 \pm 6.68$ & 0.358 \\
CD62P & $4.40 \pm 1.20$ & $4.36 \pm 1.17$ & 0.923 \\
Soluble P-selectin $(\mathrm{ng} / \mathrm{mL})(n=15)$ & $217.27 \pm 132.1$ & $207.00 \pm 83.7$ & 0.483 \\
\hline
\end{tabular}
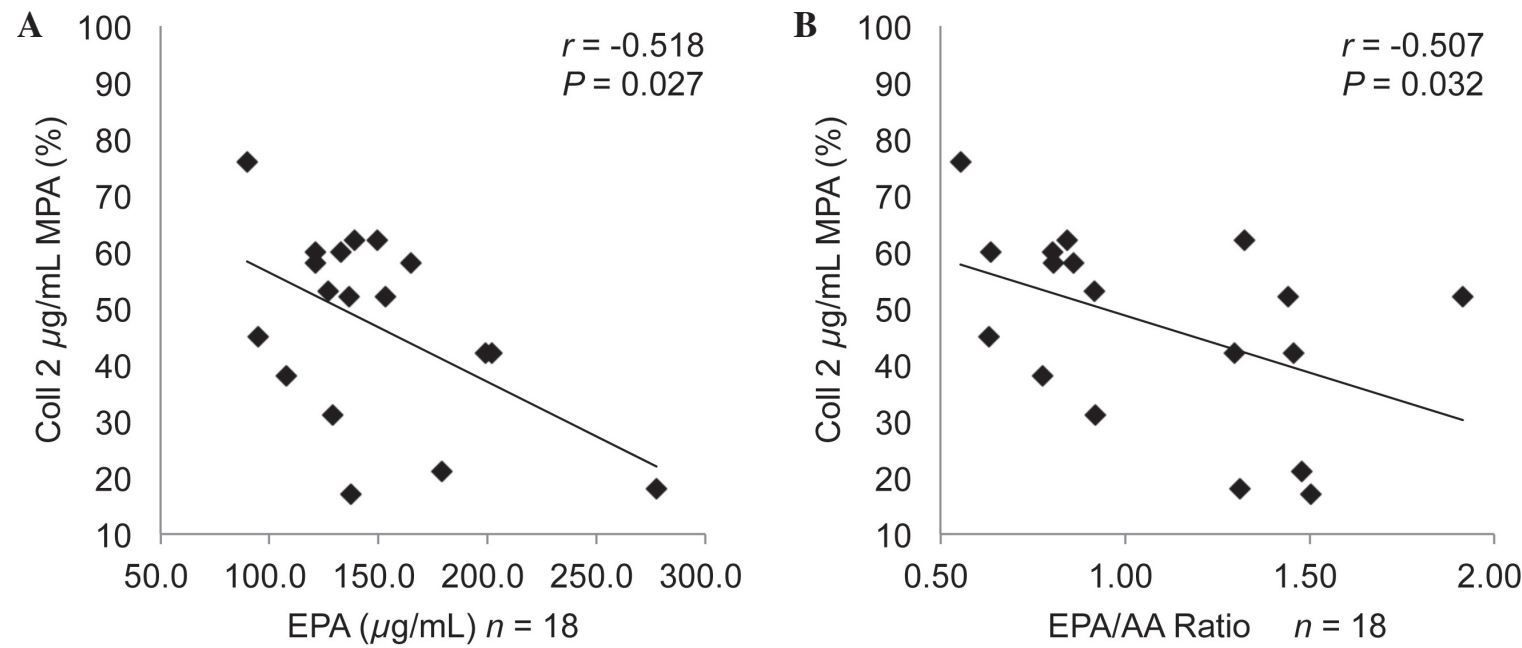

Figure 1. Correlations between collagen-induced MPA and plasma EPA concentration (A) and EPA/AA ratio (B). MPA indicates Maximum platelet aggregation; EPA, eicosapentaenoic acid ethylester; and AA, arachidonic acid.

observed in the expression of PAC-1 and CD62P on the platelet surface membranes or in the soluble P-selectin concentration (Table III).

To further evaluate the effects of EPA on platelet function, correlations between platelet aggregation and plasma fatty acid levels or the plasma EPA/AA ratio were studied. The collagen $(2 \mu \mathrm{g} / \mathrm{mL})$-induced MPA showed a significant negative correlation with the plasma EPA level $(r=-0.518, P=0.027)$ and with the EPA/AA ratio $(r=-0.507, P=0.032)$ at 12 months (Figure 1A, 1B), although no significant correlations were found at baseline $(r=-0.366, P=0.135 ; r=-0.462, P=$ 0.054 , respectively). There was no correlation between MPA and plasma AA level $(r=0.151, P=0.550)$.

We then divided the patients into 2 groups according to the median value of the plasma EPA/AA ratio of 0.92 at 12 weeks after being treated with EPA-E. There were no significant differences in baseline characteristics between the 2 groups. The EPA/AA ratio increased significantly in both the higher $(n=10)$ and lower $(n=8) \mathrm{EPA} / \mathrm{AA}$ ratio groups (baseline $0.25 \pm 0.12$ to on-treatment $0.74 \pm 0.11, P<0.0010 .52 \pm$ 0.19 to $1.36 \pm 0.29, P<0.001$ respectively) (Figure2A, 2B). The patient baseline characteristics were similar between the 2 groups (Table IV). However, after treatment, both collagenand AA-induced MPA were significantly suppressed in the higher EPA/AA ratio group $(n=10)$, while no changes were found in the lower group (Figure 3A, 3B). Multivariate linear regression analysis showed that a high EPA/AA ratio was independently and negatively associated with collagen $(2 \mu \mathrm{g} /$ $\mathrm{mL}$ )-induced MPA $(\beta=-0.639, P=0.036)$ (Table V). No suppressive effect on ADP-induced MPA was observed in either group (Figure 3C).

\section{Discussion}

In the current study, EPA-E prescribed concomitantly with ASA for secondary prevention following percutaneous coronary intervention (PCI) failed to show a clear antiplatelet effect overall. However, in patients with a high on-treatment EPA/AA ratio, EPA-E significantly suppressed platelet aggregation.

Historically, those with a high omega- 3 fatty acid intake or a high plasma EPA concentration have been reported to exhibit reduced thrombogenicity. ${ }^{10)}$ The main explanation for this reduction in thrombogenicity is that omega-3 fatty acid (or EPA) replaces AA, which is a strong agonist of platelet aggregation. Thus, an increase in EPA intake is usually followed by a decrease in AA in the body, theoretically reducing platelet 

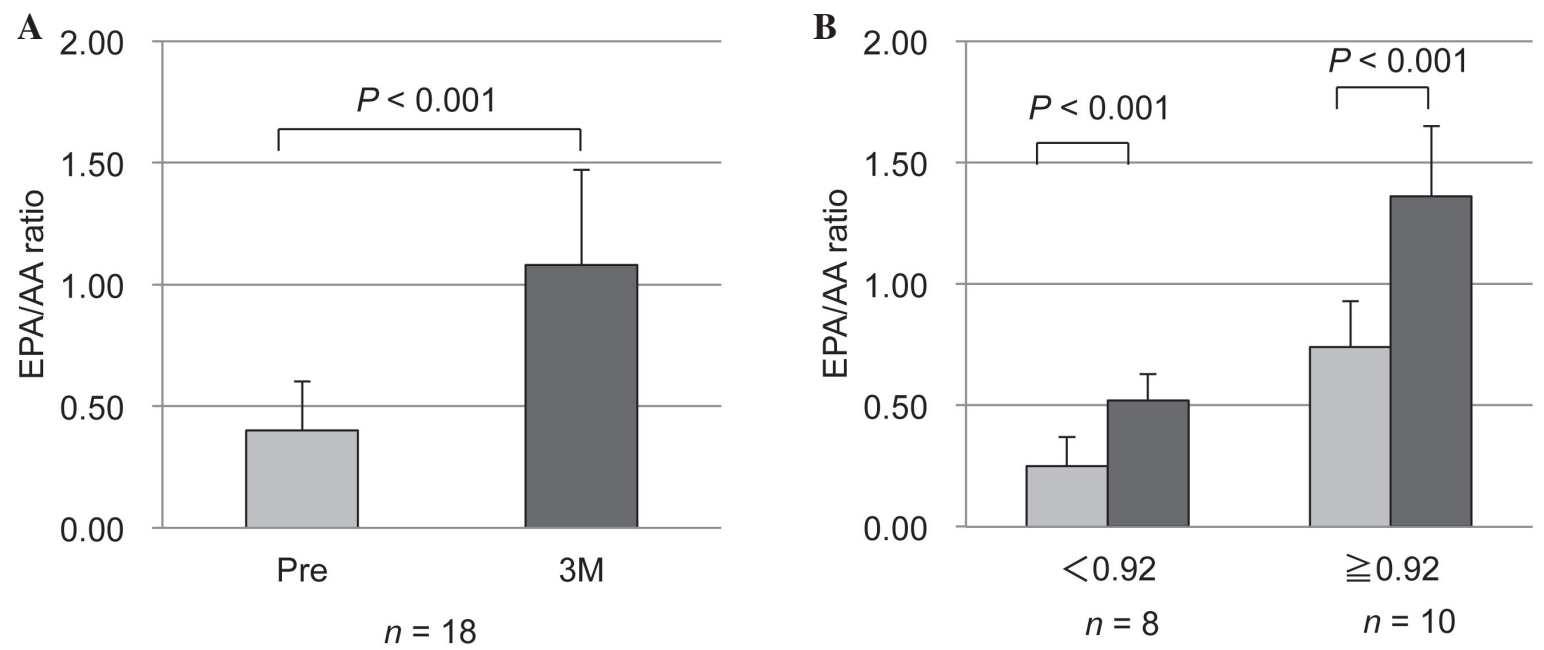

Figure 2. EPA/AA ratio before and after EPA-E administration (A), EPA/AA ratio before and after EPA-E administration which is divided into two groups according to $3 \mathrm{M}$ EPA/AA ratio $(\mathbf{B})$.

Table IV. Baseline Characteristics of the Patients Divided into 2 Groups According to the Median Value of the Plasma EPA/AA Ratio of 0.92 After EPA-E Administration

\begin{tabular}{lccc}
\hline & $\begin{array}{c}\text { EPA/AA } \geq 0.92 \\
(n=10)\end{array}$ & $\begin{array}{c}\text { EPA/AA }<0.92 \\
(n=8)\end{array}$ & $P$ \\
\hline Age (years) & $64.4 \pm 11.6$ & $62.8 \pm 5.4$ & 0.067 \\
Male & $10 / 10(100 \%)$ & $8 / 8(100 \%)$ & \\
Dyslipidemia & $10 / 10(100 \%)$ & $8 / 8(100 \%)$ & \\
Hypertension & $7 / 10(70 \%)$ & $7 / 8(87.5 \%)$ & 0.787 \\
Diabetes & $1 / 10(10 \%)$ & $4 / 8(50 \%)$ & 0.600 \\
PAD & $0 / 10(0 \%)$ & $0 / 8(0 \%)$ & \\
Smoking & $3 / 10(30 \%)$ & $5 / 8(62.5 \%)$ & 0.168 \\
\hline
\end{tabular}

Table V. Multivariate Linear Regression Analysis on Collagen $(2 \mu \mathrm{g} / \mathrm{mL})$ Induced MPA Variation

\begin{tabular}{lcc}
\hline & $\begin{array}{c}\text { Standardized } \\
\text { coefficient }(\beta)\end{array}$ & $P$ \\
\hline EPA/AA $\geq 0.92$ & -0.639 & 0.036 \\
Smoking & -0.223 & 0.392 \\
Diabetes & -0.316 & 0.230 \\
Hypertension & 0.090 & 0.708 \\
Age & 0.333 & 0.189 \\
\hline
\end{tabular}

Dependent variable: collagen $(2 \mu \mathrm{g} / \mathrm{mL})$-induced MPA.

function. Several studies have demonstrated antiplatelet effects following EPA treatment. Terano, et al studied the antiplatelet effect of EPA on healthy subjects. ${ }^{11)}$ In their study, following supplementation with EPA-E $3.6 \mathrm{~g} /$ day for 4 weeks, platelet aggregation and platelet retention were significantly suppressed. The same authors showed that the EPA content in platelet phospholipids increased markedly, whereas the AA content did not change. Suehiro, et al reported that the addition of EPA-E $1.8 \mathrm{~g} /$ day to ASA therapy significantly inhibited platelet aggregation in patients with chronic cerebral infarction. ${ }^{12)}$ Gajos, et al also showed that the addition of omega-3 polyunsaturated fatty acid to ASA and clopidogrel significantly suppressed platelet aggregation in patients undergoing PCI. ${ }^{13)}$

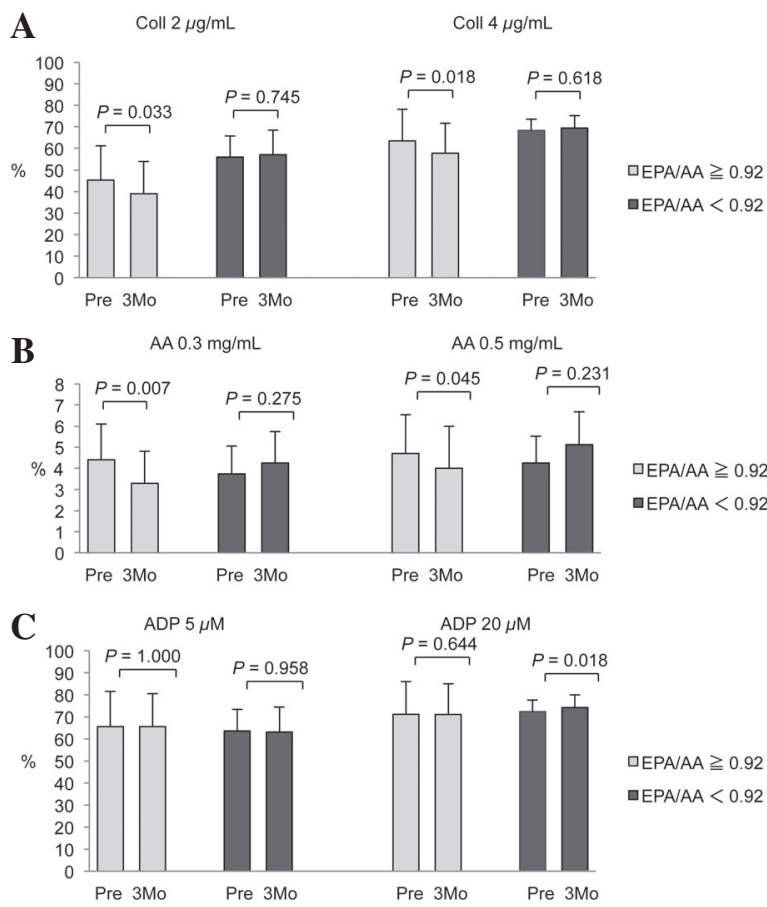

Figure 3. Comparisons of collagen-induced MPA (A), AA-induced MPA (B) and ADP-induced MPA (C) before and after EPA-E administration. ADP indicates adenosine diphosphate.

Nomura, et al. clearly showed that platelet-derived microparticles, indicating platelet activation, significantly decreased with EPA treatment in hyperlipidemic and diabetic patients. ${ }^{14)}$

Conversely, several studies failed to show any antiplatelet effects of EPA. Davi, et al. studied the add-on effect of EPA with ticlopidine on platelet function in patients with CAD ${ }^{15)}$ The aggregation responses to collagen, ADP, and AA were not altered significantly by the intake of EPA (as fish oil). Serebruany, et al. evaluated platelet function following 2 weeks of omega-3 fatty acid supplementation in patients with CAD who 
were taking ASA. ${ }^{16)}$ They used various platelet tests, including aggregometry and flow cytometry, and cartridge analyzer readings. These gave a mixture of positive and negative results, but most importantly, collagen- and AA-induced aggregation were not affected by the treatment. The lack of uniformity in study subjects and the amount of EPA supplementation, or differences between the platelet function evaluation methods may account for this disparity.

In the current study, we used several different methods for testing platelet function, including aggregometry and flow cytometry, but we could not show any positive effect of EPA-E treatment combined with ASA overall. We found a negative correlation between the on-treatment EPA/AA ratio and collagen-induced platelet aggregation, and the effects of EPA-E appeared to be insignificant in those with a low EPA/AA ratio.

The concept of EPA/AA ratio was originally introduced a long time ago, when native Inuit people in Greenland were found to have a strikingly low incidence of ischemic heart disease. The fact that they had high levels of plasma EPA and low AA concentrations suggested that their omega-3 fatty acid-rich food was implicated in this favorable outcome. ${ }^{10,17)}$ Subsequently, a number of studies reported findings indicating that a low EPA/AA ratio was related with a higher incidence of cardiovascular events in the Japanese population, ${ }^{18)}$ as well as in those with a greater number of diseased coronary vessels, ${ }^{19)}$ a higher risk of cerebrovascular accident, ${ }^{20)}$ or more visceral fat, which is a major factor in metabolic syndrome. ${ }^{21)}$

With a certain amount of EPA supplementation, the EPA/ AA ratio increases without fail as shown in the current study (Figure 2A). An increase in the EPA/AA ratio following EPA supplementation was reported to reduce cardiovascular events in the JELIS sub-study. ${ }^{8)}$ In this sub-study, the incidence of sudden cardiac death or fatal/nonfatal myocardial infarction was significantly lower in the high on-treatment EPA/AA ratio $(\geq 1.06)$ group than in the low EPA/AA ratio $(\leq 0.55)$ group. Interestingly, the current study showed similar results, revealing that a high on-treatment EPA/AA ratio is essential if EPA is to be effective in reducing platelet aggregation. The cut-off EPA/AA ratio value of 0.92 is close to that of the JELIS study. We may need to raise the EPA/AA ratio and maintain it around 1.0 in order to achieve positive effects with EPA at the clinical level. Further large-scale studies are warranted.

Limitations: The small sample size is one of the limitations of this study, and the lack of power could be related to our overall negative results. However, it is noteworthy that we were able to demonstrate a correlation between platelet function and plasma EPA concentration as well as EPA/AA ratio. Second, all the patients were men in this study, so the results may not apply to female patients. Third, the possibility of a statin-related reduction in AA metabolism has been raised. ${ }^{22)}$ However, in this study, all patients were taking statins throughout the study period; therefore, any influence on our results should be minimal. Conclusion: EPA treatment had a potential to suppress collagen-induced platelet aggregation in patients with a high plasma EPA/AA ratio. A further, large-scale study is warranted.

\section{REFERENCES}

1. Wijns W, Kolh P, Danchin N, et al. Guidelines on myocardial revascularization. Eur Heart J 2010; 31: 2501-55.
2. Smith SC Jr, Feldman TE, Hirshfeld JW Jr, et al. ACC/AHA/ SCAI 2005 guideline update for percutaneous coronary intervention: a report of the American College of Cardiology/American Heart Association Task Force on Practice Guidelines (ACC/AHA/ SCAI Writing Committee to Update the 2001 Guidelines for Percutaneous Coronary Intervention). J Am Coll Cardiol 2006; 47: e1-121.

3. Tada T, Natsuaki M, Morimoto T, et al. Duration of dual antiplatelet therapy and long-term clinical outcome after coronary drugeluting stent implantation: landmark analyses from the CREDOKyoto PCI/CABG Registry Cohort-2. Circ Cardiovasc Interv 2012; 5: 381-91.

4. Kimura T, Morimoto T, Nakagawa Y, et al. Antiplatelet therapy and long-term clinical outcome after sirolimus-eluting stent implantation: 5-year outcome of the j-Cypher registry. Cardiovasc Interv Ther 2012; 27: 181-8.

5. Robinson JG, Stone NJ. Antiatherosclerotic and antithrombotic effects of omega-3 fatty acids. Am J Cardiol 2006; 98: 39i-49i. (Review)

6. Iso $\mathrm{H}$, Kobayashi M, Ishihara J, et al. Intake of fish and $\mathrm{n} 3$ fatty acids and risk of coronary heart disease among Japanese: the Japan Public Health Center-Based (JPHC) Study Cohort I. Circulation 2006; 113: 195-202.

7. Yokoyama M, Origasa H, Matsuzaki M, et al. Effects of eicosapentaenoic acid on major coronary events in hypercholesterolaemic patients (JELIS): a randomised open-label, blinded endpoint analysis. Lancet 2007; 369: 1090-8.

8. Matsuzaki M, Yokoyama M, Saito Y, et al. Incremental effects of eicosapentaenoic acid on cardiovascular events in statin-treated patients with coronary artery disease. Circ J 2009; 73: 1283-90.

9. Tagawa T, Hirooka Y, Shimokawa H, et al. Long-term treatment with eicosapentaenoic acid improves exercise-induced vasodilation in patients with coronary artery disease. Hypertens Res 2002; 25: 823-9.

10. Dyerberg J, Bang HO, Stoffersen E, Moncada S, Vane JR. Eicosapentaenoic acid and prevention of thrombosis and atherosclerosis? Lancet 1978; 2 : 117-9.

11. Terano T, Hirai A, Hamazaki T, et al. Effect of oral administration of highly purified eicosapentaenoic acid on platelet function, blood viscosity and red cell deformability in healthy human subjects. Atherosclerosis 1983; 46: 321-31.

12. Suehiro A, Higasa S, Ueda M, Oura Y, Kakishita E. Combination effect of eicosapentaenoic acid and platelet suppressive agents on platelets. Curr Ther Res 1994; 55: 653-9.

13. Gajos G, Rostoff P, Undas A, Piwowarska W. Effects of polyunsaturated omega-3 fatty acids on responsiveness to dual antiplatelet therapy in patients undergoing percutaneous coronary intervention: the OMEGA-PCI (OMEGA-3 fatty acids after PCI to modify responsiveness to dual antiplatelet therapy) study. J Am Coll Cardiol 2010; 55: 1671-8.

14. Nomura S, Inami N, Shouzu A, et al. The effects of pitavastatin, eicosapentaenoic acid and combined therapy on platelet-derived microparticles and adiponectin in hyperlipidemic, diabetic patients. Platelets 2009; 20: 16-22.

15. Davi G, Belvedere M, Catalano I, et al. Platelet function during ticlopidine and eicosapentaenoic acid administration in patients with coronary heart disease. Platelets 1990; 1: 81-4.

16. Serebruany VL, Miller M, Pokov AN, et al. Early impact of prescription Omega-3 fatty acids on platelet biomarkers in patients with coronary artery disease and hypertriglyceridemia. Cardiology 2011; 118: 187-94.

17. Bang HO, Dyerberg J, Nielsen AB. Plasma lipid and lipoprotein pattern in Greenlandic West-coast Eskimos. Lancet 1971; 1: 11435.

18. Domei T, Yokoi H, Kuramitsu S, et al. Ratio of serum n-3 to n-6 polyunsaturated fatty acids and the incidence of major adverse cardiac events in patients undergoing percutaneous coronary intervention. Circ J 2012; 76: 423-9.

19. Hayakawa S, Yoshikawa D, Ishii H, et al. Association of plasma $\omega-3$ to $\omega-6$ polyunsaturated fatty acid ratio with complexity of cor- 
onary artery lesion. Intern Med 2012; 51: 1009-14.

20. Ikeya Y, Fukuyama N, Kitajima W, Ogushi Y, Mori H. Comparison of eicosapentaenoic acid concentrations in plasma between patients with ischemic stroke and control subjects. Nutrition 2013 29: $127-31$

21. Inoue K, Kishida K, Hirata A, Funahashi T, Shimomura I. Low se- rum eicosapentaenoic acid/ arachidonic acid ratio in male subjects with visceral obesity. Nutr Metab (Lond) 2013; 10: 25.

22. Harris JI, Hibbeln JR, Mackey RH, Muldoon MF. Statin treatment alters serum n-3 and n-6 fatty acids in hypercholesterolemic patients. Prostaglandins Leukot Essent Fatty Acids 2004; 71: 263-9. 